\title{
Neumonia lipoidea. Causa infrecuente de infiltrados pulmonares
}

\author{
MARÍA J. SOLER-SEMPERE*, JORGE OLEA-SOTO**, JUAN A. GALLEGO-SÁNCHEZ*** \\ y MARÍA DEL M. DÍAZ-GARCÍA****
}

\section{Lipoid pneumonia. An infrequent cause of pulmonary infiltrates}

The presence of pulmonary infiltrates is a frequent finding that includes a large differential diagnosis based on many occasions in the clinical history. Among them, exogenous lipoid pneumonia represents a rare entity and a high index of suspicion is necessary to reach its diagnosis and prevent its progression. In these cases, an adequate clinical context and a CT with opacities and low density areas are highly suggestive of the disease. We present a case of exogenous lipoid pneumonia secondary to the continued use of oily substances at the nasal level, due to his antecedents and the diagnostic possibilities after the CT findings, histological confirmation was required.

Key words: Pneumonia, lipid; diagnosis, differential; tomography X-Ray computed.

\section{Resumen}

La presencia de infiltrados pulmonares es un hallazgo frecuente que incluye un amplio diagnóstico diferencial basado en muchas ocasiones en la historia clínica. Entre ellas, la neumonía lipoidea exógena representa una entidad poco frecuente y es preciso un elevado indice de sospecha para alcanzar su diagnóstico y evitar su progresión. En estos casos, un contexto clínico adecuado y una TC con opacidades y áreas de baja densidad pueden ser altamente sugestivos de la enfermedad. Se presenta un caso de neumonía lipoidea exógena secundaria a la utilización continuada de sustancias oleosas intranasal, que debido a los antecedentes del paciente y a las posibilidades diagnósticas tras los hallazgos de la $T C$, precisó confirmación histológica.

Palabras clave: Neumonía lipoidea; diagnóstico diferencial; tomografía computarizada.

\section{Introducción}

Las opacidades pulmonares en vidrio deslustrado son un hallazgo muy inespecífico. Su presencia obliga al clínico a realizar un amplio diagnóstico diferencial basado en la anamnesis, los síntomas, la exploración física y los exámenes complementarios. En muchas ocasiones, la historia clínica detallada del paciente va a permitir orientar la sospecha diagnóstica, evitando exploraciones innecesarias. Sin embargo, existen casos en los que, a pesar de la sospecha clínica y los hallazgos en las exploraciones complementarias, es necesaria la confirmación histológica.

Presentamos el caso de un paciente en el que sus antecedentes clínicos y la anamnesis detallada contribuyeron a establecer la causa de los infiltrados radiológicos.

\section{Caso clínico}

Un hombre de 72 años fue remitido a la consulta de neumología tras el hallazgo de infiltrados pulmonares persistentes en la radiografía de

\footnotetext{
* Sección de Neumología.

** Unidad de Hospitalización a Domicilio.

*** Servicio de Radiodiagnóstico.

**** Medicina Familiar y Comunitaria Hospital General Universitario de Elche, Alicante, España.
} 
tórax. Exfumador de 80 paquetes/año, entre sus antecedentes destacaba la exéresis de la epiglotis por un carcinoma infiltrante $y$, posteriormente se le practicó una resección de la glándula salivar palatina por carcinoma epitelial. Años después fue intervenido por un carcinoma adenoide quístico en nasofaringe y en el seno maxilar con varias recidivas posteriores que fueron tratadas con cirugía y radioterapia. Para aliviar los síntomas de sequedad nasal y nasofaríngea como consecuencia de las intervenciones y a la radioterapia recibida, el paciente se administraba vaselina nasal. En sus estudios de control, comenzaron a apreciarse unos infiltrados pulmonares bilaterales persistentes a pesar de recibir diversas pautas de antibioterapia y esteroides sistémicos. El paciente refería disnea leve con escasa tos y expectoración. En la auscultación pulmonar presentaba crepitantes bilaterales de predominio derecho. En la tomografía computarizada (TC) torácica se apreciaban opacidades basales de predominio izquierdo, algunas de ellas mostraban zonas de menor densidad (Figuras 1 y 2) y otras un patrón en empedrado de predominio izquierdo (Figura 3). Aunque la TC puede revelar áreas de densidad grasa inferiores a - $30 \mathrm{UH}$ dentro de las áreas de consolidación y de los nódulos, un hallazgo diagnóstico de neumonía lipoidea, la presencia de inflamación superpuesta puede ser un factor de confusión aumentando la densidad de los componentes grasos y haciendo estos menos evidentes ${ }^{1}$.

Estos hallazgos junto con la presencia de áreas en vidrio deslustrado establece un amplio diagnóstico diferencial con la infección por Pneumo-

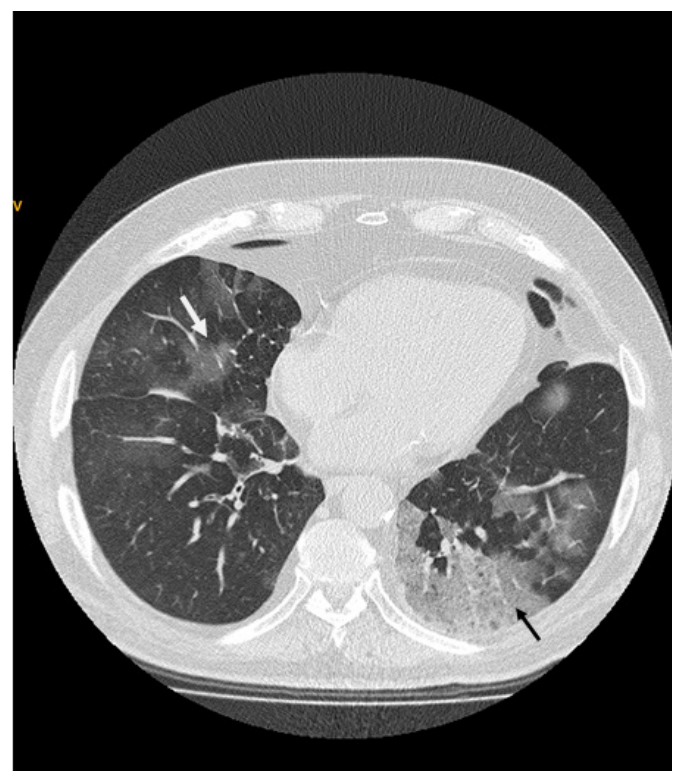

Figura 1. Opacidades basales de predominio izquierdo con áreas de menor atenuación (Flechas).

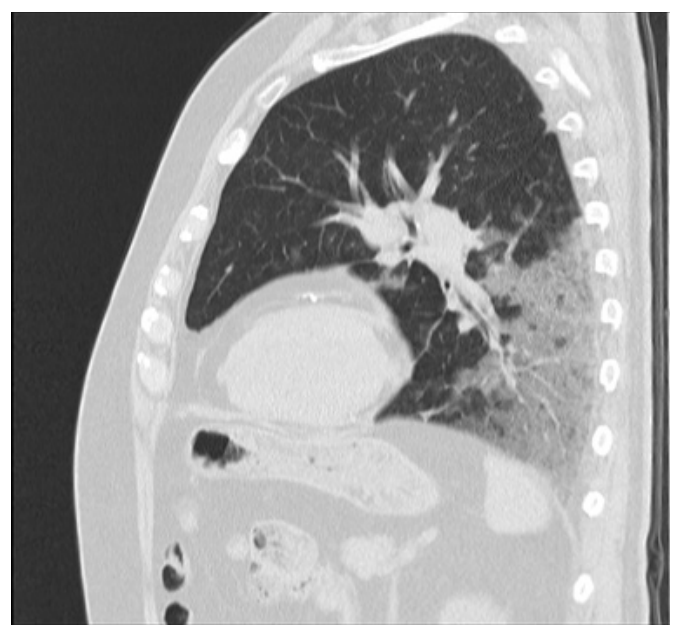

Figura 2. Opacidades basales de predominio izquierdo.

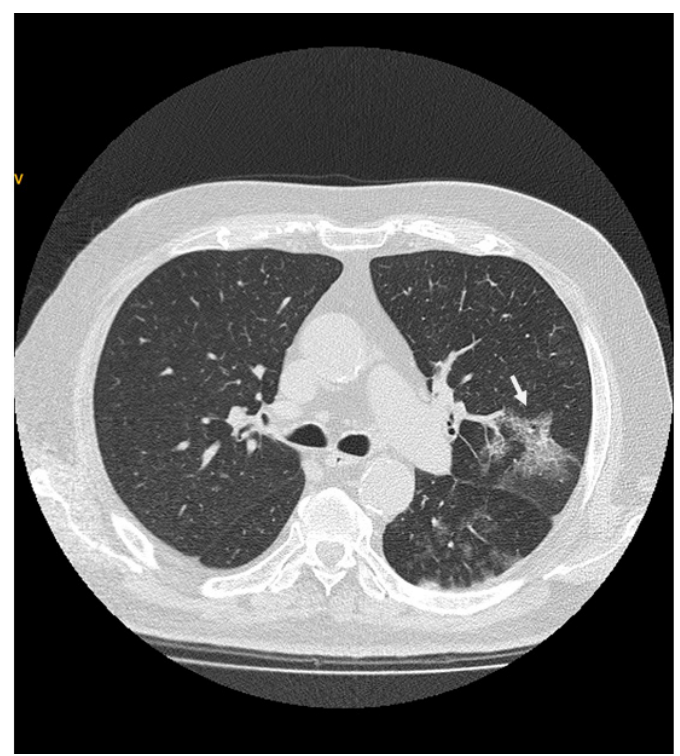

Figura 3. Opacidad en empedrado en lóbulo superior izquierdo (Flecha).

cistis Jirovecii, el adenocarcinoma mucinoso, la proteinosis alveolar, la sarcoidosis, la neumonía intersticial inespecífica, la neumonía organizada, la neumonía lipoidea exógena, el síndrome de distrés respiratorio y la hemorragia pulmonar ${ }^{2}$. En nuestro caso tras correlacionar los hallazgos radiológicos con la situación clínica del paciente y sus antecedentes se valoraron como principales opciones diagnósticas el adenocarcinoma mucinoso, la sarcoidosis, la neumonía intersticial inespecífica y la neumonía lipoidea.

Ante estos hallazgos, se realizó una broncoscopia, que no puso de manifiesto lesiones endobronquiales. Se realizó un lavado broncoalveolar (BAL) y se tomaron muestras de tejido a través de biopsia transbronquial en el lóbulo inferior izquierdo. En la muestra del BAL se observaron 
macrófagos con inclusiones lipídicas, y el examen anatomopatológico de las muestras de tejido mostró una arquitectura pulmonar distorsionada a expensas de una expansión de los septos ocupados casi en su totalidad por celularidad histiocitoide con amplios citoplasmas multivacuolados y presencia de histiocitos espumosos intraalveolares, compatible con una neumonía lipoidea (Figura 4). A pesar de la suspensión del uso de vaselina intranasal, en el seguimiento radiológico se apreció una persistencia de los infiltrados pulmonares.

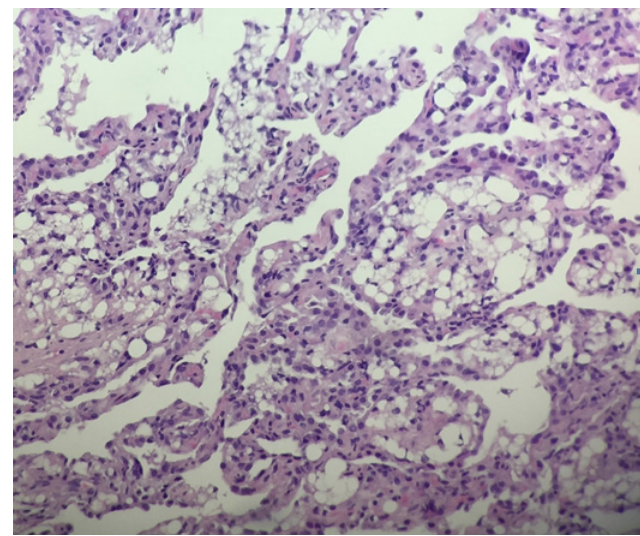

Figura 4. Biopsia transbronquial. Distorsión del parénquima pulmonar debido a un engrosamiento septal por la presencia de celularidad multivacuolada de hábito histiocítico.

\section{Discusión}

La neumonía lipoidea exógena es una entidad infrecuente, causada por la aspiración de material graso y su acumulación a nivel intraalveolar, como consecuencia del uso de sustancias oleosas en la vía aérea ${ }^{3}$. Descrita por primera vez por Laughlen en 1925, al encontrar el hallazgo de gotas oleosas en un estudio de necropsia tras la aspiración de aceite mineral suministrado como laxante o gotas nasales ${ }^{4}$. De origen exógeno o endógeno, se trata de una entidad infrecuente, producida en la mayor parte de los casos, por la inhalación repetida de material graso debido al uso de aceites minerales en pulverizaciones o gotas nasales 5 . También se ha relacionado con la aspiración accidental de vaselina utilizada en sondajes nasogástricos ${ }^{6,7,8}$. En cambio, las formas endógenas se desarrollan al escapar de los alvéolos los lípidos del tejido pulmonar en relación con una obstrucción debida a un proceso neoplásico. Resultando menos frecuente su asociación con la proteinosis alveolar, embolia grasa o las formas idiopáticas 9 .

Aunque se han descrito formas agudas en casos de aspiración masiva de material lipídico ${ }^{10}$, la forma más habitual de presentación es la crónica, generalmente como resultado de episodios repetidos de aspiración o de inhalación de grasa animal o vegetal. En estos casos, los aceites aspirados al llegar a los alvéolos son fagocitados por los macrófagos lo que provocará la formación de pequeñas vacuolas. Éstos, incapaces de metabolizarlos, rompen su pared dando lugar a una liberación de ácidos grasos provocando una respuesta inflamatoria a cuerpo extraño y una migración hacia el septo interlobulillar ${ }^{5}$.

Las manifestaciones clínicas varían en función de la forma de presentación. Con frecuencia, se trata de un proceso infiltrativo crónico como consecuencia de episodios de aspiración continuados. En estos casos, los síntomas suelen ser inespecíficos como disnea, tos improductiva e incluso puede cursar de forma asintomática ${ }^{5,8}$. Sin embargo, en los casos de aspiración masiva de lubricantes oleosos o de aceite de parafina cursarán con aparición de disnea brusca, tos, fiebre e incluso insuficiencia respiratoria ${ }^{8,11}$.

Los hallazgos radiológicos consisten en el aumento de densidad radiológico con patrón en vidrio deslustrado, la consolidación, el patrón en empedrado, y la presencia de nódulos o masas ${ }^{5}$, de modo que pueden simular otras enfermedades pulmonares ${ }^{2}$. En consecuencia, se debe establecer un diagnóstico diferencial con otras entidades como la infección por Pneumocistis Jirovecii, el adenocarcinoma mucinoso, la proteinosis alveolar, la sarcoidosis, la neumonía intersticial inespecífica, la neumonía organizada, el síndrome de distrés respiratorio y la hemorragia pulmonar ${ }^{2}$.

Las alteraciones en la TC que se describen con más frecuencia son la presencia de opacidades y consolidaciones con áreas de atenuación en rango de tejido graso (entre $-30 \mathrm{y}-150 \mathrm{UH}$ ), en vidrio deslustrado peribronquiales y de predominio basal pueden apoyar el diagnóstico ${ }^{12}$. También se han descrito en forma de masa con zonas de densidad grasa y con patrón en empedrado (patrón intersticial superpuesto a opacidades en vidrio deslustrado). En un contexto clínico adecuado, hay autores que sugieren que la presencia de estas opacidades podría resultar altamente sugestivas de la enfermedad y evitar la realización de exploraciones invasivas ${ }^{3,9,12}$. Sin embargo, cuando se presenta como lesiones nodulares o masas8 obliga en estos casos a establecer un diagnóstico diferencial con una lesión neoformativa, como sucedió en nuestro paciente.

La utilidad de la resonancia magnética es desconocida para estos casos, a pesar de que puede mostrar la presencia de grasa como señal hiperintensa en secuencias T1, T2 o cambio químico en fase opuesta. La TC con contraste es considerada la mejor herramienta de imagen para caracterizar las lesiones y determinar la presencia de un componente graso. En el caso de la Tomo- 
grafía computarizada-Tomografía por emisión de positrones (TC-PET), las lesiones con aspecto de masa crónica con frecuencia son positivas en la PET y se pueden confundir con neoplasias si no se busca específicamente grasa en la $\mathrm{TC}^{13}$.

En los casos en los que existe un claro antecedente aspirativo, se puede establecer un diagnóstico inicial de alta probabilidad basado en los datos clínicos y radiológicos ${ }^{6}$. En cambio, en las formas crónicas, las manifestaciones clínicas pueden ser inespecíficas ocasionando demoras diagnósticas ${ }^{6,14}$, y en determinados casos resulta imprescindible incrementar el índice de sospecha, especialmente en casos de edad avanzada y con factores de riesgo, como sucede en pacientes laringectomizados en los que es frecuente el uso de vaselina para facilitar el cambio de cánula ${ }^{3}$.

Aunque los estudios de imagen pueden aportar información de indudable valor diagnóstico, pueden presentar limitaciones. En cualquier caso, para establecer un diagnóstico de certeza se debe demostrar la presencia de macrófagos cargados de lípidos en muestras de lavado broncoalveolar o de tejido pulmonar ${ }^{6,7,14}$.

El tratamiento de la neumonía lipoidea se basa en evitar la exposición al agente causal y tratar las complicaciones asociadas. En nuestro caso a pesar de la suspensión del uso de vaselina intranasal, en el seguimiento radiológico se apreció una persistencia de los infiltrados. El uso de esteroides sistémicos es controvertido y se ha reservado para casos graves de presentación agu$\mathrm{da}^{1,8}$. Pensamos que nuestro paciente, además de seguir las recomendaciones médicas para aliviar los síntomas locales como consecuencia de las intervenciones en las fosas nasales y paranasales, presentaba una alteración de los mecanismos de defensa locales como la ausencia de epiglotis, circunstancia que pudo contribuir al desarrollo de la entidad.

En definitiva, la neumonía lipoidea exógena es una entidad potencialmente evitable en determinados casos, y que debe tenerse en cuenta en el diagnóstico diferencial de los infiltrados pulmonares persistentes, siendo necesaria un elevado índice de sospecha basada en la historia clínica de los pacientes, sobre todo si presentan factores de riesgo.

\section{Bibliografía}

1.- BETANCOURT S, MARTINEZ-JIMENEZ S, ROSSI SE, TRUONG MT, CARRILLO J, ERASMUS JJ. Lipoid pneumonia: spectrum of clinical radiologic manifestations. AJR 2010; 194: 103-109.

2.- ROSSI SE, ERASMUS JJ, VOLPACCHIO M, FRANQUET T, CASTIGLIONI T, MCADAMS P. "Crazy-Paving" pattern at thin-section CT of the lungs: Radiologic-Pathologic Overview. RadioGraphics 2003; 23: $1509-19$.
3.- GARCÍA-LATORRE, R, RODRÍGUEZ-DÍAZ R, BARRIOS-BARRETO D, AYALA-CARBONERO A, GARCÍA-GÓMEZ MI, GOROSPE-SARASÚA L. Hallazgos radiológicos de la neumonía lipoidea exógena en pacientes laringuectomizados. Arch Bronconeumol 2015; 51: 36-9.

4.- LAUGHLEN GF. Studies on pneumonia following nasopharyngeal injections of oil. Amer J Path 1925; 1: 407-11.

5.- ALIAGA F, CHERNILO S, FERNÁNDEZ C, VALENZUELA H, RODRÍGUEZ JC. Exogenous lipoid pneumonia. Report of three cases. Rev Med Chile 2017; 145: $1495-9$.

6.- ROMERO-CANDEIRA S, MARTÍN-SERRANO C, PALAU-BENAVENT M, TOBÍAS-FERRER J, ARANDA-LÓPEZ I, ESCORTELL-MAYOR E. Neumonía Lipoidea aspirativa. Arch Bronconeumol 1989; 25: 28-30.

7.- BERNABEU MORA R, MENÉNDEZ MARTÍNEZ P, ABELLÁN MARTÍNEZ MC, POLO GARCÍA LA, LORENZO CRUZ M, SÁNCHEZ GASCÓN F. Neumonía lipoidea aguda debida a la aspiración accidental de vaselina utilizada en un sondaje nasogástrico. Arch Bronconeumol 2000; 36: 485-7.

8.- ALAMINOS-GARCÍA P, COLODRO-RUIZ A, MENDUIÑA-GUILLÉN MJ, BÁNEZ-SÁNCHEZ F, PÉREZ-CHICA G. Neumonía lipoidea exógena. Presentación de un nuevo caso. An Med Interna 2005; 22: 283-4.

9.- BYERLEY JS, HERNÁNDEZ ML, LEIGH MW, ANTOON JW. Clinical approach to endogenous lipoid pneumonia. Clin Respir J 2016; 10: 259-63.

10.- JARA-PALOMARES L, MÁRQUEZ-MARTÍN E, RODRÍGUEZ-PORTAL A, TALLÓN-AGUILAR R, DOMINGUEZ-PETIT A, MARTÍN-JUAN J. Neumonía lipoidea exógena aguda por aspiración accidental de gasolina. Neumosur 2007; 19: 84-7.

11.- ÁLVAREZ-CORDOVÉS MM, MIRPURI-MIRPURI PG, ROCHA-CABRERA E, PÉREZ-MONJE A. Lipoid pneumonia: presentation of a case. Semergen 2013; 39: 110-12.

12.- MACHIORI E, ZANETTI G, MAURO-MANO C, HOCHHEGGER B. Exogenous lipoid pneumonia. Clinical and radiological manifestations. Respir Med 2011; 105 : 659-66.

13.- GURNEY JW, WINER-MURAM HT, ROSADO-DECHRISTENSON ML,MONAMMED TH, .ABBOT GF, MAIE MH. Especialidades en imagen. TACAR de pulmón. Lippincott Williams \& Wilkins and Amirsys. Marbán 2013.

14.- HADDA V, KHILNANI GC. Lipoid pneumonia: an overview. Expert Rev Res Med 2010; 4: 799-807. 393-9.

\footnotetext{
Correspondencia a:

Dra. María J. Soler-Sempere

Sección de Neumología

Hospital General Universitario de Elche

Alicante, España.

Email: majosoler1@hotmail.com
} 